\title{
Gao Xingjian - Entre le chinois et le français
}

\section{Simona Gallo}

\section{(2) OpenEdition}

\section{Journals}

\section{Édition électronique}

URL : https://journals.openedition.org/genesis/3018

DOI : 10.4000/genesis.3018

ISSN : 2268-1590

\section{Éditeur :}

Presses universitaires de Paris Sorbonne (PUPS), Société internationale de génétique artistique littéraire et scientifique (SIGALES)

\section{Édition imprimée}

Date de publication : 4 juin 2018

Pagination : 115-120

ISBN : 979-10-231-0604-6

ISSN : 1167-5101

\section{Référence électronique}

Simona Gallo, "Gao Xingjian - Entre le chinois et le français », Genesis [En ligne], 46 | 2018, mis en ligne le 01 juin 2019, consulté le 27 octobre 2021. URL : http://journals.openedition.org/genesis/3018 ; DOI : https://doi.org/10.4000/genesis.3018 


\title{
Entre le chinois et le français Entretiens avec Gao Xingjian (Prix Nobel)
}

\author{
Propos recueillis par Simona Gallo
}

Gao Xingjian 高行健 (Ganzhou, 1940) - déclaré persona non grata 1 en Chine continentale depuis 1990 et citoyen français à partir de 1998 - est écrivain et artiste peintre. Après avoir abandonné la Chine, il s'est installé en France et il a choisi de se consacrer totalement à la quête d'une liberté dans la création; son ouvre littéraire a été couronnée par le prix Nobel de littérature en 2000. Gao Xingjian a toujours revendiqué une position «à la marge ${ }^{2}$, qui se situe au-delà de l'identité nationale. En effet, il est aujourd'hui, selon les mots de Pascale Casanova, un écrivain bilingue «international 3 », qui aime se définir comme un «citoyen du monde ${ }^{4}$.

Les propos ici rapportés proviennent de trois entretiens que nous avons réalisés avec Gao Xingjian à diverses occasions. Le premier, le 21 février 2013, a été enregistré à Paris, en chinois; les citations ici proposées ont été traduites par nos soins depuis la version italienne. Le deuxième, daté du 10 avril 2015, a été mené en langue française à Paris également. Le troisième et dernier a été enregistré à Milan le 21 septembre 2016, en langue française.

\section{Penser et écrire entre deux langues}

Simona Gallo - Bonjour Monsieur Gao. Vous êtes un artiste et écrivain bilingue, et donc vous vivez toujours dans une double dimension linguistique, celle du chinois et celle du français. Comment décrivez-vous aujourd'hui votre rapport à ces deux langues?

Gao Xingjian - Je vis à Paris depuis vingt-sept ans déjà. Je n'ai que très rarement l'occasion de parler chinois, sauf avec mon épouse; souvent on parle aussi français. Dans les rencontres de tous les jours et dans la vie quotidienne, y compris la vie professionnelle et artistique, on parle plutôt français. C'est très pratique pour moi, cela devient une langue courante sans traduction dans mon esprit.

J'écris aussi en deux langues : ma première commande en langue française m'a été passée par le ministère de la Culture français, pour une pièce de théâtre. C'était au début des années
1990 : à cette époque, j’avais déjà quitté la Chine où tous mes livres étaient censurés. Voilà, j'ai pensé : si j'écris encore en chinois, il n'y a pas de public, il n'y a pas la possibilité d'être mis en scène. Alors pourquoi écrire en chinois quand on vit en Occident? Cela m'intéressait de me lancer dans cette aventure d'écrire dans une autre langue qui n'était pas ma langue maternelle : c'était très difficile. Je dois vous avouer que pour écrire une première phrase... C'était une longue phrase, en français bien structuré. Et aussi musicalement - il faut bien jouer avec la musicalité - c'était «à la française». J'ai mis

1. Toutes ses œuvres sont encore interdites en Chine continentale. 2. Gilbert Fong C. F., «Un esprit libre à la marge : l'art et la vision de Gao Xingjian», dans Noël Dutrait (dir.), L'Écriture romanesque et théâtrale de Gao Xingjian, Paris, Éditions du Seuil, 2006, p. 205.

3. Pascale Casanova, La République mondiale des lettres, Paris, Éditions du Seuil, [1999] 2008, p. 224.

4. Simona Gallo 與高行健對談 [entretien avec Gao Xingjian], 21 février 2013. 
trois jours et trois nuits pour écrire cette longue phrase. Car si on écrit en français, il faut une belle écriture en français, mais ce n'est pas ma langue maternelle. Et donc, avec plusieurs dictionnaires à mes côtés, il fallait réécrire, reconstruire en permanence, c'était une aventure. Mais une fois qu'on y est entré, on y va sans passer par la traduction mentale. Dès le début, les phrases, les idées viennent directement en français. C'est pourquoi, si j'écris en français, je pense en français. Si j'écris en chinois, je pense en chinois, sans passer par l'interprétation ou par la traduction. Et parce que les deux langues sont tellement éloignées l'une de l'autre... Le chinois est très souple, il n'y a même pas de conjugaisons, pas de modes ni de temps. Un caractère chinois peut être à la fois un nom, un adjectif, un verbe, un adverbe... tout est possible. Cela dépend de la structure, de comment on dispose l'ordre des éléments de la phrase. Alors qu'en français tout est très logique, très rationnel, mais aussi très structuré. Et $\mathrm{j}$ 'ai déjà écrit cinq pièces, directement en français, et aussi certains poèmes, directement en français. Mais si je dois publier en chinois, ce n'est pas la peine de faire traduire mes propres œuvres écrites directement en français par un traducteur. Pour une belle écriture en chinois, je dois fabriquer une autre œuvre. Ce n'est pas comme chez Beckett; je ne peux pas confronter son écriture en anglais et en français, même dans En attendant Godot, par exemple, je ne sais pas quelle est la différence entre les deux versions, comment cela se passe pour lui : pour moi, il s'agit d'une autre écriture. J'ai déjà l'original en français : le mode de vie, la façon de s'exprimer, c'est très français, très occidental, très loin des habitudes de la pensée chinoise. Mais si j'écris en chinois, pour avoir une belle écriture, il faut inventer. Certes, on ne peut pas inventer n'importe quoi. En chinois, le monde de la grammaire n'existe que depuis une centaine d'années. C'est l'œuvre d'un prêtre chinois, qui est venu en France, qui a étudié le latin, et puis, pour aider les Occidentaux à apprendre le chinois plus facilement, il s'est servi de la grammaire du latin, du français, pour expliquer la langue chinoise. C'était donc la première grammaire du chinois, mais plutôt occidentalisée.

\section{S.G. - Le Mashiwentong [馬氏文通], c'est cela?}

G.X. - Oui, Ma Jianzhong [馬建忠] a inventé la grammaire chinoise, le Mashiwentong [馬氏文通], pour aider les Occidentaux à comprendre cette pensée. Cela veut dire que dans la pensée chinoise, cette rationalité, cette logique n'est pas apparente : c'est là la grande différence. Et pourtant, je pense que j'ai réuni les deux dans mon écriture. Parce que je suis très conscient du côté chinois, dans la poésie, dans mes pièces de théâtre, dans mes romans. Souvent je m'amuse à faire cela. Quelquefois, on peut dire que c'est très chinois, quelquefois on peut dire que c'est très occidental, très bien défini, structuré. Et même quand j'exprime mon esthétique j'ai écrit pas mal sur l'esthétique - je travaille sur la précision. C'est tellement défini, tellement net, tellement précis, que cela n' appartient pas au chinois normalement. [...] je regrette que l'on ait occidentalisé la langue chinoise moderne. Cela a appauvri la richesse, le charme de cette langue. Ma Jianzhong a introduit dans le chinois moderne, par exemple...

\section{S.G. - ... singulier et pluriel, ou bien masculin et féminin...}

G.X. - Tout à fait, et puis les verbes, les adjectifs, les noms. Mais pour la vraie langue chinoise zhongwen [中文], la couleur verte $l \ddot{\text { [ }}$ [綠], cela peut être un verbe : chunfeng you lü jiang liang'an [春風又綠江兩岸] : «le printemps a verdi le sud de la rivière». En français, cela donne deux mots, d'une part le verbe «verdir» lï [綠] et d'autre part l'adjectif «vert», lü de [綠的]5.

Un autre exemple : Ta lian dou lü le [他臉都綠了]. Cela veut dire «il est en colère», «il a eu un choc», mais c'est une expression tellement vivante en chinois. Il y en a beaucoup d'autres, comme bian shuo bian chi [邊說邊吃] [on parle tout en mangeant], en chinois c'est shuozhe chizhe [說著 吃著], ce qui correspond à des formes anglaises en «-ing».

\section{S.G. - C'est donc le présent progressif...}

G.X. - Voilà, c'est cela, «être en train de», «en faisant quelque chose», et donc «tout en parlant on mange», shuozhe chizhe [說著吃著]. Mais dans mon écriture littéraire, artistique, je n'utilise pas cette forme. En effet, la

5. Dans la grammaire du chinois moderne, 的 $\boldsymbol{d e}$ est une marque de détermination nominale que l'on trouve dans des syntagmes nominaux, entre le déterminant et le déterminé, comme 我的朋友 wo de pengyou («mon ami»), 她穿的衣服 ta chuan de yifu («la robe qu'elle porte»). La présence de 的 $\boldsymbol{d} \boldsymbol{e}$ est généralement nécessaire entre l'adjectif et le nom, si l'adjectif est dissyllabique ou bien modifié par un adverbe, comme 很好的朋友 hen hao de pengyou («très bon ami»). 
langue chinoise est très souple, et je défends cette souplesse, qui est à l'origine du chinois; si on veut écrire en bon chinois, c'est comme cela. Dans la vie moderne, cette langue a vieilli, elle ne suffit plus. Il faut créer. Et comment créer, voilà le problème. Introduire grammaticalement les langues occidentales dans cette langue-là? Non, il faut trouver ce qui est déjà dans la langue, en germe. Alors j'invente. Mais j'invente d'une autre façon, sans passer par la grammaire des langues occidentales. Je dois me fonder sur cette langue-là. Pour moi, c'est l'émotion qui compte.

S.G. - Tout à l'heure vous avez évoqué votre écriture plurilingue : vous écrivez en chinois, et également en français?

G.X. - Oui, j'écris en deux langues, surtout pour le théâtre ${ }^{6}$. Mais pour une nouvelle version en chinois, ce que je fais est une invention. Je dois faire disparaître toutes les traces de la traduction, comme si c'était une version originale : c'est une nouvelle création. La version chinoise est une réécriture de la même pièce de théâtre. Il ne s'agit en aucun cas de traduction. Quand je traduis, je rencontre des difficultés remarquables. Il est très dur pour moi de traduire d'une langue à une autre : réaliser une bonne traduction est vraiment difficile. Comme je suis moi-même le traducteur, je ne peux pas me permettre de traduire librement, parce que dans l'acte de traduire il n'y a pas cette liberté-là. Mais en même temps, comme je suis l'auteur, je m'autorise cette liberté. Je m'explique : j'ai écrit cinq drames en français, qui n'ont absolument rien à voir avec la Chine. Ce que j'ai écrit concerne seulement l'Occident contemporain. Écrits en chinois, ces drames n'ont pas le même contexte, parce que les contextes sociaux et culturels en France et en Chine sont trop éloignés. J'ai écrit sur l'Occident contemporain, sur la vie en Occident aujourd'hui, et ensuite j'ai essayé d'écrire en un bon chinois. Je veux créer une langue chinoise qui soit réelle, c'est seulement ainsi que cela sera du bon chinois. Le chinois que je veux n'est pas une langue toute faite, elle doit rechercher un sentiment nouveau; grâce à un chinois actuel, je veux réussir à communiquer l'émotion de la vie réelle dans un contexte occidental. C'est sans doute un défi, mais c'est aussi très intéressant. Pour moi, c'est vraiment ainsi. Pour cela je dois de toute façon écouter ce que j'écris. Que ce soit du français ou du chinois, je dois écouter la langue.
S.G. - Il s'agit précisément de ces cinq pièces réécrites : Au bord de la vie, Le somnambule, Quatre quatuors pour un week-end, Le quêteur de la mort et Ballade nocturne. Votre travail d'autotraduction ou bien de réécriture est particulièrement intéressant. Mais pourquoi réécrire une pièce dans une autre langue?

G.X. - Le premier critère réside dans le fait que la pièce de théâtre est jouée sur scène. Ce n'est donc pas de la lecture classique, ce n'est pas intellectuel, il faut vraiment un langage vivant. Bien sûr, il y a aussi une valeur littéraire, mais ce n'est pas comme le chinois classique : le chinois archaïque est très concis. Pour s'adresser à la sensibilité de l'écoute, il faut vraiment faire attention à l'intonation, aux tons, et tout doit être très vivant. Cela peut provoquer immédiatement une réaction du public, et donc c'est encore plus difficile de traduire. En chinois, il y a beaucoup d'expressions toutes faites dans la langue archaïque. On appelle cela wenyan wen [文言文], cela veut dire «écriture des lettrés», une langue que personne ne comprend à l'oral, sauf les lettrés. J'évite ces expressions toutes faites de la langue chinoise archaïque, mais je dois inventer, créer une expression très concise, vivante et sensible à l'écoute.

S.G. - Dans l'essai Le chinois moderne et l'écriture littéraire, vous écriviez que pour obtenir une belle écriture vous deviez. vous enregistrer et puis vous écouter. Est-ce que vous faites cela encore aujourd'hui?

G.X. - Oui, je pense qu'il y a un filtre très important pour l'écriture. L'écriture, oui, c'est écrire à la main ou bien à la machine. Mais l'essentiel pour une langue, c'est aussi une quête. D'après moi, je pense qu'on parle la langue avec une voix, pas par l'écriture. L'écriture cela vient après, cela laisse une trace. Mais la sensibilité directe, c'est par la voix, les intonations, et aussi la compréhension immédiate pour celui qui écoute ce texte-là. Pour moi, enregistrer mes textes me sert à savoir si on peut écouter ce texte-là, si cela va ou non, si c'est bon ou non, sensible ou non, impressionnant ou non. Ce n'est pas une recherche de structure de l'écriture, cela, c'est autre chose.

6. Extrait de l'entretien enregistré le 10 avril 2015 à Paris. 


\section{S.G. - Pensez-vous que la connaissance de la langue fran- çaise a influencé votre écriture en chinois?}

G.X. - Pas directement, parce que les deux langues sont très éloignées. Mais le français influence ma façon de penser, alors que le chinois est trop souple, et souvent il n'a pas cette précision de la pensée. Si on écrit pour exprimer une pensée, si on fait de la théorie, si on veut vraiment définir quelque chose, je pense, le français est magnifique, tandis que le chinois n'est pas si précis. C'est pourquoi je peux dire que la langue française m'a beaucoup aidé à réfléchir sur mes écrits théoriques. Par exemple, j'ai aussi écrit beaucoup d'articles sur l'esthétique, surtout l'esthétique de la création fondée sur mes propres expériences. Et après, cela devient une écriture quelquefois philosophique, esthétique et très théorique. Dans ce cas-là, on peut voir l'influence de la façon de penser caractéristique de la langue française. C'est pourquoi il est si facile d'être traduit par les traducteurs en d'autres langues occidentales, parce que tout y est tellement logique, tellement défini, tellement précis. Pour s'exprimer, cette précision est très importante, ce n'est pas comme la poésie.

S.G. - Et donc on pourrait dire que vous rédigez vos essais théoriques en chinois, mais pas «à la façon chinoise ${ }^{7}$ »?

G.X. - Pour exprimer mes réflexions théoriques je ne peux pas m'exprimer à la façon chinoise. Tous ces essais-là sont très bien définis, et cette façon d'écrire est plutôt très à l'occidentale.

S.G. - En revenant sur le thème de la création plurilingue, votre dernier cinépoème, Le Deuil de la beauté, mêle différentes langues (chinois, français et anglais) et différents langages artistiques. Voulez-vous commenter ce choix?

G.X. - Mon dernier film, Le Deuil de la beauté 8 , est en trois langues, mais il n'y a pas de sous-titrage. Je pense que cela dérange, quand on voit le film et qu'il y a un sous-titrage. Et aussi parce que je suis également peintre : toutes les images doivent être parfaites. S'il y a un sous-titrage c'est bien dommage, voilà pourquoi je m'exprime en trois langues. $\mathrm{Si}$ on comprend une de ces langues, on peut comprendre le film sans passer par la traduction ni par le sous-titrage.

\section{Vivre entre deux cultures}

S.G. - Que reste-t-il de la culture chinoise dans votre création, dans vos cuvres?

G.X. - Vous voulez dire maintenant?

S.G. - Oui, maintenant.

G.X. - Cela dépend de ce que l'on considère. Si l'on considère mes peintures, ou mon public, alors c'est une situation très variée. Par exemple, les pièces de théâtre que j'ai écrites sur l'Occident n'ont rien à voir avec la Chine, elles sont marquées par une perception très occidentale, des sentiments très occidentaux.

Mais si on les analyse attentivement, on peut y trouver un point de vue chinois sous-jacent : c'est le cas de Au bord de la vie, qui est le monologue intérieur d'une femme, mais exprimant exclusivement les sentiments d'une femme occidentale. Vous me demandez si ma manière d'écrire est marquée par la Chine? Je dois dire que oui. De quelle façon? Effectivement, c'est par l'usage de la troisième personne du singulier «il », qui dépasse le soi, qui est comme un troisième œil pour observer à l'intérieur du moi. D'habitude, ce n'est pas ainsi que l'on exprime cette intériorité en Occident; d'habitude on utilise la première personne, «je». Mais utiliser la troisième personne pour manifester l'activité de la conscience, aucun écrivain occidental ne l'avait fait, personne avant moi n'avait parcouru ce chemin. Et ce choix est lié à la culture chinoise. En regardant bien, même si j'écris sur l'Occident, on voit la culture chinoise en toile de fond. C'est naturel. Un autre auteur n'écrirait pas ainsi,

7. Gao Xingjian commence à publier des essais théoriques et critiques sur l'art et la littérature, en Chine, dans les années 1970. On compte aujourd'hui plus d'une centaine d'essais en langue chinoise et six recueils, que nous mentionnons en bibliographie, dont certains ont été traduits en français par Noël Dutrait.

8. Gao a réalisé le film Le Deuil de la beauté en 2013. Le titre du film reprend celui du recueil publié en 2012 aux Éditions Simoncini. «Le titre vient d'un long poème, une réflexion sur notre temps. Le sens du beau a disparu, remplacé par la mode, la consommation, la même dans le monde entier. Le goût de la pub a remplacé l'art. Le film est un hommage à la richesse du patrimoine universel. » (Gao Xingjian, interview dans Libération, décembre 2013). 
il n'utiliserait pas comme moi cette troisième personne, cela ne pourrait même pas lui venir à l'esprit. Si l'on examine en profondeur mon écriture, on découvre qu'il y a le contexte d'une culture chinoise qu'un écrivain occidental ne pourrait jamais vraiment créer.

\section{S.G. - Ça, c'est votre signature?}

G.X. - Dans mes écrits c'est très évident : il y a la culture chinoise et en même temps la culture occidentale, dongxi [東西]. Et pour moi, l'écriture n'est pas fondée sur la culture chinoise, pour introduire l'occidentale. Non, ce n'est pas cela. Pour moi, il n'y a pas une culture qui est plus importante qu'une autre. Pas plus qu'il n'existe une culture qui est dominante, qui est assimilée, qui est l'identité. Cette question ne se pose pas pour moi. Tout est assimilé dans ma pensée, dans mon écriture. J'ai une pensée chinoise, j'ai une pensée occidentale. Je les connais assez bien toutes les deux. Et entre les deux, il n'y a pas d'opposition entre une pensée dominante et une pensée assimilée. Non. Mais si on analyse tout cela, bien sûr, il y a évidemment une pensée d'origine chinoise, il y a même une façon de penser, une méthode... shenzhi you yi ge fangfalun [甚至有一個方法論]. Et en ce qui concerne l'Occident aussi, parce que j' ai appris le français. Le français, grammaticalement, est très structuré. La rationalité y est tellement marquée. Au contraire, la culture chinoise n'est pas dans la rationalité, il n'y a pas de forme.

\section{S.G. - C'est du pragmatisme.}

G.X. - Oui, le concept de rationalité n'existe pas en chinois. De même, la logique est très peu importante. Parce qu'il y a une autre façon de penser. Cela vient d'abord de Laozi (Lao Tseu) [老子] et Zhuangzi ${ }^{9}$ [莊子], et ensuite du Chanzong 10 [禪宗]. C'est tout à fait une autre pensée : on peut dire que c'est une pensée très orientale, très spéciale. Difficile à comprendre pour les Occidentaux. Comment peut-on passer d'un point à l'autre ? $\mathrm{Si}$ «oui » devient «non», si «noir» devient «blanc»... heibai [黑白] [noir et blanc], shifei [是非] [vrai et faux], duicuo [對錯] [correct et faux]. Tout cela devient l'autre. C'est la non-dualité permanente ! Au contraire, la pensée occidentale est bien marquée par...

S.G. - Des dichotomies?
G.X. - Voilà. Dichotomies, antinomies. Et aussi la dialectique marquée par la pensée d'Hegel, et aussi er lü bei fan [二律 背反] [les antinomies]... C'est tout un système fondé sur la rationalité, à la base de la pensée occidentale, et bien marqué dans la langue aussi.

\section{S.G. - C'est un problème de la langue écrite.}

G.X. - Tout à fait. Si l'on examine attentivement la langue parlée, nous découvrirons que de nombreuses phrases à l'européenne sont fondamentalement inutiles dans le dialogue.

S.G. - Finalement, comment vous placez-vous entre deux grandes cultures si différentes? Quelle est votre position?

G.X. - Quelle est ma position désormais? Je me définis comme «citoyen du monde». Je dis que j'ai eu trois vies : la première en Chine, la deuxième en exil, la troisième en France.

Après avoir gagné le prix Nobel, à partir de l'année 2000 j'ai commencé à voyager partout, j'ai fait le tour du monde. J'ai voyagé dans tous les pays dont j'ai accepté l'invitation, et j'ai reçu vraiment beaucoup d'invitations de partout dans le monde. Ma vie est ainsi : aujourd'hui je suis là, demain je serai là-bas... Je vais partout. C'est pourquoi je dis que je suis un citoyen du monde, et c'est la pure vérité. C'est vraiment ainsi!

Maintenant j'écris mes œuvres en chinois ou en français, mais elles ne sont pas nécessairement adressées au lecteur français. Je m'adresse au monde entier. De fait, mes œuvres ont déjà été traduites dans quarante et une langues. Quarante et une! Et je suis allé partout. Mes peintures ont été exposées dans tous les coins de la Terre. Ma peinture n'est pas chinoise, et le public ne la voit pas comme telle, même s'il s'agit de peintures à l'encre. Mes films aussi sont allés partout, tout comme mes œuvres théâtrales sont mises en scène chaque année dans différents pays. Le public les apprécie, le spectateur ne pense pas qu'il s'agit d'une œuvre chinoise, tout le monde les comprend, même les plus idiots. Bien sûr, j'ai créé aussi de nombreuses pièces de théâtre en langue chinoise, mais beaucoup d'entre elles transcendent

9. Premiers philosophes taoïstes de la tradition chinoise

10. Le bouddhisme de l'école chinoise Chan, ou Zen. 
le contexte culturel. Alors comment voulez-vous m'assigner une identité, désormais? Je pense être un citoyen du monde.

\section{S.G. - Votre lieu de vie est le monde! \\ G.X. - Oui, c'est le monde !}

\section{Bibliographie}

Casanova Pascale, La République mondiale des lettres, Paris, Éditions du Seuil, [1999] 2008

FONG C.F. Gilbert, «Un esprit libre à la marge : l'art et la vision de Gao Xingjian », dans Noël Dutrait (dir.), L'Écriture romanesque et théâtrale de Gao Xingjian, Paris, Éditions du Seuil, 2006, p. 205-224.

GALLO Simona 與高行健對談 [Entretien avec Gao Xingjian], 21 février 2013.

GALLO Simona et GAO Xingjian 高行健, Entretien à Paris, 10 avril 2015.
GALLO Simona et GAO Xingjian 高行健, Entretien à Milan, 21 septembre 2016.

GAO Xingjian 高行健, Xiandai xiaoshuo jiqiao chutan 現代小說技巧 初探 [Premier essai sur les techniques du roman moderne], Canton, Huacheng, 1981

GAO Xingjian 高行健, Dui yizhong xiandai xiju de zhuiqiu 對一種現代 戲劇的追求 [Recherches pour un théâtre moderne], Pékin, Zhongguo xiju chubanshe, 1988.

GAO Xingjian 高行健, Mei you zhuyi 沒有主義 [Ne pas avoir de -ismes], Hong Kong, Tiandi tushu chuban gongsi, 1996.

GAO Xingjian 高行健, «Le chinois moderne et l'écriture littéraire», dans Le Témoignage de la littérature, Paris, Éditions du Seuil, 2004, p. 79-107. GAO Xingiian 高行健, Ling yi zhong meixue 另一種美學 [Pour une autre esthétique], Taïwan, Linking books, 2001.

GAO Xingjian 高行健, Lun chuangzuo 論創作 [De la création], Taïwan, Linking books; Hong Kong, Mingbao yuekan chubanshe; Singapour, Xinjiapo qingnian chubanshe shuju, 2008.

GAO Xingjian 高行健, Lun xiju 論戲劇 [Du théâtre], Taïwan, Linking books, 2010.

GAO Xingjian 高行健, Ziyou yu wenxue自由與文學 [Liberté et littérature], Taïwan, Linking books, 2014

SimONA GALLO est docteur en Études linguistiques, littéraires et interculturelles à l’Università degli Studi de Milan (Italie). Ses domaines de recherche concernent la littérature chinoise et sinophone, l'écriture plurilingue et la traduction culturelle. Elle fait partie de l'Associazione Italiana per gli Studi Cinesi (AISC), de l'European Association for Chinese Studies (EACS) et du Contemporary Asia Research Center (CARC). Elle est actuellement professeur de langue, littérature et culture chinoise auprès de différentes universités italiennes, et elle et elle a publié des articles concernant l'esthétique transculturelle, l'intertextualité et l'écriture critique de Gao Xingjian. simona.gallo@unimi.it 